\title{
Sintonia Ótima do Controlador PID em um Sistema AVR Utilizando Busca Tabu
}

\author{
Frank Wesley Rodrigues* Clivaldo Silva Araújo* \\ Yuri P. M. Rodriguez ${ }^{*}$ Kaíque R. A. Albuquerque* \\ Jonathan J. P. Moura * Thommas K. S. Flores* \\ * Departamento de Engenharia Elétrica, Universidade Federal da \\ Paraíba,PB, (e-mail: frank.rodrigues@cear.ufpb.br, \\ clivaldo@cear.ufpb.br,molina.rodriguez@cear.ufpb.br, \\ kaique.albuquerque@cear.ufpb.br,jonathan.moura@cear.ufpb.br, \\ thommas.flores@cear.ufpb.br).
}

\begin{abstract}
This work has as objective the study the optimum tuning of the proportionalintegral-derivative controller (PID) in an Automatic Voltage Regulator (AVR) system performed using the Tabu Search optimization method. It is possible to observe in detail the use of the proposed method to efficiently search for PID parameters in order to provide the greatest control of terminal voltage in an AVR system. The efficiency of the algorithm is analyzed for response to the unitary step obtaining its characteristics through the response in time. The values found with the proposed method were compared with results determined by Genetic Algorithm (GA) and Particle Swarm Optimization (PSO).

Resumo: Este trabalho tem como objetivo o estudo da sintonia ótima do controlador proporcional-integral-derivativo (PID) em um sistema Regulador Automático de Tensão (AVR - Automatic Voltalge Regulator) realizada através do método de otimização de Busca Tabu. É possível observar com detalhes a utilização do método proposto para busca eficiente dos parâmetros do PID com o objetivo de proporcionar o maior controle da tensão terminal em um sistema AVR. A eficácia do algoritmo é analisada para resposta ao degrau unitário obtendo suas características através da resposta no tempo. Os valores encontrados com o método proposto foram comparados com resultados determinados pela técnica de Algoritmo Genético (GA - Genetic Algorithm) e de Otimização por Enxame de Partícula (PSO - Particle Swarm Optimization).
\end{abstract}

Keywords: Automatic Voltage Regulator; PID Controller; Tabu Search Optimization. Palavras-chaves: Regulador Automático de Tensão; Controle PID; Otimização por Busca Tabu.

\section{INTRODUÇÃO}

Em um sistema elétrico de potência manter constante o nível de tensão nominal e controlar a potência reativa recebida ou fornecida à rede elétrica é um dos principais tipo de problema relacionado com a qualidade de energia, segurança e confiabilidade da rede. Sabemos que, enquanto a quantidade instantânea de potência reativa em um sistema de potência depende do nível de tensão, a quantidade instantânea de potência ativa em uma rede elétrica depende da frequência do sistema. Essas potências devem ser mantidas estáveis nas condições de estado estacionário do sistema de potência. Por outro lado, os dispositivos conectados à rede foram considerados para o nível de tensão desejado, denominado valor nominal, para um funcionamento adequado e seguro. Se o valor de tensão disponível for alterado, a eficiência do equipamento conectado ao sistema de energia será consideravelmente afetada e a expectativa de vida útil diminuirá. Para reduzir as perdas de transmissão é necessário manter o equilíbrio

* O presente trabalho foi realizado com apoio da Fundação de Apoio à Pesquisa do Estado da Paraíba (FAPESQ) - Termo $n^{\circ} 280 \backslash 18$. da potência reativa para o controle do valor de tensão do sistema de potência. Para resolver esses problemas o AVR é adicionado aos geradores síncronos (Elgerd, 1982).

Muitas técnicas de controle não lineares e lineares podem ser aplicadas no ajuste do controle do AVR. Dentre essas técnicas as de controle de autoajuste pode ser separada das outras, devido ao seu influente desempenho de controle, que é quase independente das variações dos valores do sistema. Os trabalhos de controle de autoajuste para AVR teve início com Swidenbank et al. (1999) e Finch et al. (1999). Porém, a técnica clássica de autoajuste inclui muitos cálculos algébricos e podem se tornar inadequados em algumas situação de trabalho, como especificações de carga não-lineares e em condições de trabalho variáveis (Gözde et al., 2017). Tendo isso em vista, a utilização de métodos de autoajuste baseados em algoritmos heurísticos começou a ser utilizada para implementar o sistema AVR, especialmente o controlador PID. Gaing (2004) realizou uma comparação do ajuste do PID pela técnica de Otimização por Enxame de Partícula (PSO - Particle Swarm Optimization) e por Algoritmo Genético (Genetic Algorithm). Farouk and Bingqi (2012) implementaram lógica 
Fuzzy para o ajuste do PID, enquanto Lahcene et al. (2017) utilizou á técnica de Simulated Annealing.

Neste trabalho é utilizado a técnica de Busca Tabu para o ajuste do controlador PID no sistema AVR. Essa técnica é uma boa alternativa para encontrar o mínimo ou máximo global do espaço busca, além de proporcionar um baixo tempo de cálculo (Areerak and Sujitjorn, 2002).

O trabalho esta dividido da seguinte forma: a modelagem do sistema AVR é verificado na Seção 2. Na Seção 3 é apresentada uma introdução sobre Busca Tabu. A aplicação da técnica de Busca Tabu para ajuste do PID em um sistema AVR na Seção 4. Os resultados e simulação na Seção 5 e por fim, na Seção 6 é realizado a conclusão final.

\section{MODELAGEM DO SISTEMA AVR E PID}

O Regulador Automático de Tensão de um gerador síncrono tem como principal função o controle da tensão terminal e da energia reativa gerada. Desta forma, devido a este influenciar diretamente no campo magnético do gerador, o AVR está relacionado a estudos da estabilidade dinâmica das máquinas síncronas (Kundur et al., 1994).

$\mathrm{Na}$ Figura (1) temos a representação do sistema em diagrama de blocos, onde é constituído basicamente pelo controlador PID, AVR, excitação, gerador síncrono e por um sensor.

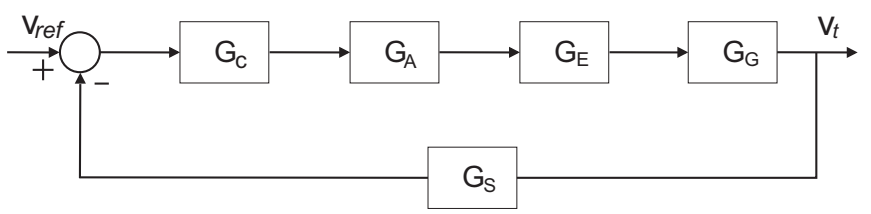

Figura 1. Diagrama de bloco do sistema.

Onde:

$G_{C}$ : função de transferência do PID;

$G_{A}$ : função de transferência do AVR;

$G_{E}$ : função de transferência da excitação;

$G_{G}$ : função de transferência do gerador;

$G_{S}$ : função de transferência do sensor;

$V_{\text {ref }}:$ tensão de referência;

$V_{t}:$ tensão terminal

\subsection{Controle PID}

O controlador PID é utilizado para melhorar a resposta dinâmica do sistema bem como para eliminar ou reduzir o erro de regime permanente (Gaing, 2004). O controle proporcional $\left(K_{p}\right)$ produz o sinal de saída que é proporcional à amplitude do erro, o controle derivativo $\left(K_{d}\right)$ adiciona um zero na função de transferência de circuito aberto da planta melhorando a sua resposta transitória, enquanto o controlador integral $\left(K_{i}\right)$ proporciona adição de um polo na origem, aumentando a ordem do sistema e reduzindo o erro de regime permanente. A função de transferência do controlador PID é observada em (1).

$$
P I D(s)=K_{p}+\frac{K_{i}}{s}+s K_{d}
$$

\subsection{Amplificador}

O modelo do amplificador utilizado é representado por um ganho $K_{A}$ e uma constante de tempo $T_{A}$ e sua função de transferência é dada por (2).

$$
\frac{V_{R}(s)}{V_{e}(s)}=\frac{K_{A}}{1+s T_{A}}
$$

$\mathrm{O}$ valor de $K_{A}$ varia entre 10 e 400, enquanto a constante $T_{A}$ é muito pequena variando entre 0,02 a $0,1 \mathrm{~s}$.

\subsection{Excitação}

A Equação (3), refere-se a função de transferência da excitação e é representada pelo ganho $K_{E}$ e pela constante de tempo $T_{E}$.

$$
\frac{V_{F}(s)}{V_{R}(s)}=\frac{K_{E}}{1+s T_{E}}
$$

Onde, o valor de $K_{E}$ esta na faixa de 1 a 400 e o valor da constante $T_{E}$ entre 0,4 a $1,0 \mathrm{~s}$.

\subsection{Gerador sincrono}

A função de transferência que retrata o modelo do gerador síncrono utilizada neste trabalho, relaciona a tensão terminal do gerador com a tensão de campo e pode ser observada em (4).

$$
\frac{V_{t}(t)}{V_{F}(t)}=\frac{K_{G}}{1+s T_{G}}
$$

Onde, $K_{G}$ é um ganho que varia entre 0,7 e 1,$0 ; T_{G}$ é a constante de tempo e possui valores entre 1,0 e 2,0 s.

\subsection{Sensor}

O sensor é modelado por uma função de transferência de primeira ordem, observada em (5):

$$
\frac{V_{s}(t)}{V_{F}(t)}=\frac{K_{R}}{1+s T_{R}}
$$

A constante $K_{R}$ corresponde ao ganho e seu valor esta na faixa de 1,0 a 10; $T_{R}$ é uma constante de tempo e possui um valor muito pequeno que esta na faixa de 0,001 a 0,06 s.

\section{BUSCA TABU}

Busca Tabu consiste na técnica de otimização para solução de problemas combinacionais. É de característica adaptativa e possui a habilidade de fazer uso de outros métodos, tais como algoritmos de soluções lineares e heurísticos (Glover, 1989).

Foi proposto inicialmente por Glover (1986) e Hansen (1986) baseado em diversos procedimentos combinatórios 
aplicados a solução de problemas de cobertura não linear, procedimentos esses introduzidos em Glover (1977). Com base em estruturas de memória, surge como uma técnica para guiar algoritmos de busca local ao longo do seu espaço de busca por soluções que vão além de seus ótimos locais (Gaspar-Cunha et al., 2012).

\subsection{Visão geral}

A Busca Tabu parte de uma solução inicial e move-se através de seu espaço de busca para um vizinho próximo, este vizinho é uma solução válida dentro de um espaço de soluções geradas ao redor da vizinhança da solução estipulada inicialmente. A execução da Busca Tabu além da premissa de inicialização de uma solução inicial, o movimento para a exploração de outras soluções necessita também de definições como: critério de escolha da próxima solução válida; regras de proibição (tabu); tamanho de memória para armazenar as proibições (lista tabu); critérios para aspiração. Além destes componentes básicos outras técnicas são empregadas para tornar o algoritmo mais sofisticado, como por exemplo o trabalho exposto em Thainiam (2018) que apresenta as técnicas de diversificação e intensificação. Essas técnicas auxiliam na exploração do espaço de busca e tornam a execução do algoritmo mais eficiente.

Das técnicas básicas é valido comentar sobre aspiração e lista tabu. A lista é usada para conter todos os movimentos que são proibidos, estes representam soluções locais já visitadas e portanto para que seja mantida a lógica de nunca se limitar a soluções locais os movimentos são restritos para os mesmos. A lista tabu na sua representação original possui memória de curto prazo, ou seja, movimentos antes proibidos com o tempo vão sendo eliminados da lista tabu para dar espaço a novos movimentos serem proibidos. $\mathrm{Na}$ ocorrência de uma dessas soluções locais seja candidata a ser a solução global do problema entra o critério de aspiração, que promove a solução como sendo válida dado seu caráter de possível global. Este é só um exemplo do que pode representar o critério de aspiração mas em sua essência é a habilidade de retirar de uma solução seu status de tabu, este critério é conhecido como aspiração por objetivo global (Gaspar-Cunha et al., 2012).

\subsection{Algoritmo}

O Algoritmo é como o proposto por Glover (1989), e parte de uma solução inicial $\left(s_{0}\right)$, onde esta é tida como a melhor solução local $(s)$ e global $\left(s_{g}\right)$ até o momento, com a resposta de sua função objetivo $(f())$ como o primeiro critério de aspiração $(A)$ por objetivo global. A lista tabu é iniciada como um conjunto vazio $(T \leftarrow \emptyset)$ e é definido também o número máximo de iterações $(\max I t)$ que representa o critério de parada do algoritmo.

A cada iteração $(I t)$ é criada uma vizinhança $(V)$ ao redor da melhor solução onde cada vizinho é definido como sendo uma solução deslocada por um fator de movimento $(m)$ da solução atual. Para cada elemento da vizinhança é avaliado o valor da função objetivo $(f())$, o melhor vizinho torna-se então $s^{\prime}$. Sendo o valor da sua $f()$ melhor que $A$, este torna-se $s_{g}$ e o novo critério de aspiração $A$. Caso não seja solução global é avaliado seu pertencimento a lista $T$, em pertencendo este valor é descartado e uma nova iteração é calculada tendo como solução central para criação da nova vizinhança utilizando-se o valor anterior de $s$. Não pertencendo a $T$, a solução atual torna-se o centro para calcular a nova vizinhança, como também passa a pertencer à $T$. Passado-se um determinado número de iterações a lista tabu é esvaziada. Ao fim, quando o critério de parada é alcançado a solução global $\left(s_{g}\right)$ é apresentada junto de seu valor de aptidão $f\left(s_{g}\right)$. O procedimento aqui comentado é exposto na Figura 2.

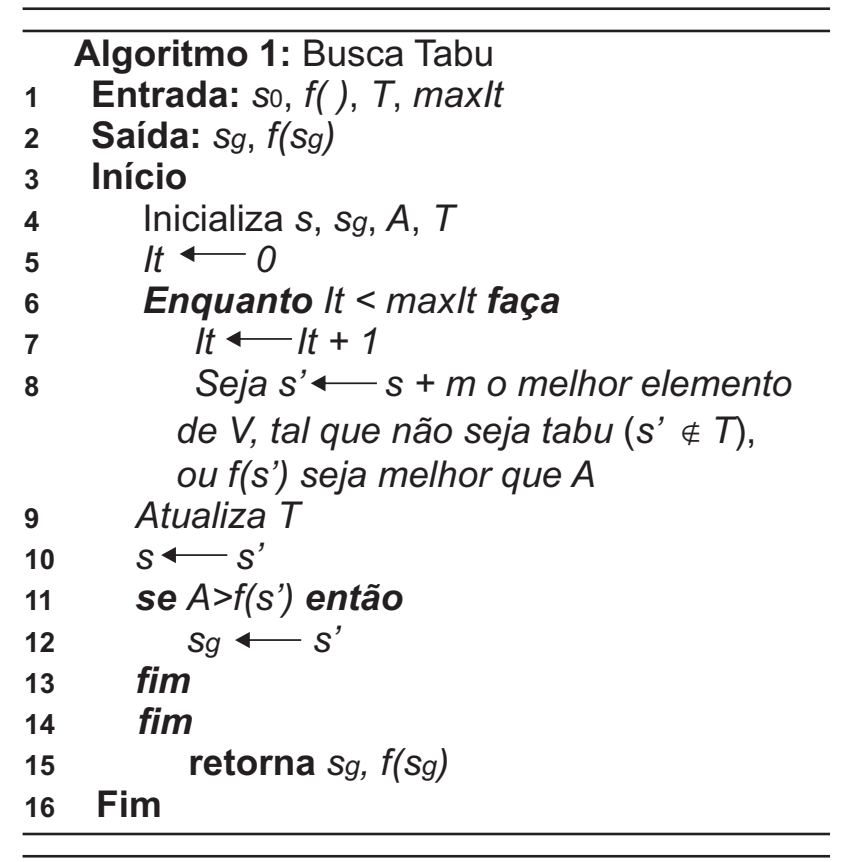

Figura 2. Algoritmo da Busca Tabu

\section{IMPLEMENTAÇÃO DO CONTROLADOR PID POR BUSCA TABU}

Neste trabalho utilizou a técnica de Busca Tabu para determinar os valores do controlador PID que proporcione uma melhora na resposta transitória do sistema AVR em um gerador síncrono. O algoritmo de Busca Tabu foi aplicado principalmente para determinar os parâmetros ótimos do controlador $K_{p}, K_{i}$ e $K_{d}$, de forma que o sistema controlado possa proporcionar uma boa saída para a resposta ao degrau.

\subsection{Sistema AVR com PID}

Baseado nas funções de transferência descrita na Seção 2 e conforme a Figura 1 é possível determinar o modelo do sistema AVR empregado no trabalho. Na Figura 3 é possível verificar o diagrama de bloco do sistema AVR com PID, os valores dos ganhos e das constantes de tempo de cada parâmetro é observado na Tabela 1.

\subsection{Função Objetivo}

Baseando-se em quatro parâmetros da resposta no tempo do sistema: máximo sobressinal $\left(M_{p}\right)$, erro de regime permanente $\left(E_{s s}\right)$, tempo de acomodação $\left(t_{s}\right)$ e tempo de subida $\left(t_{r}\right)$ é analisado a eficiência do processo de 


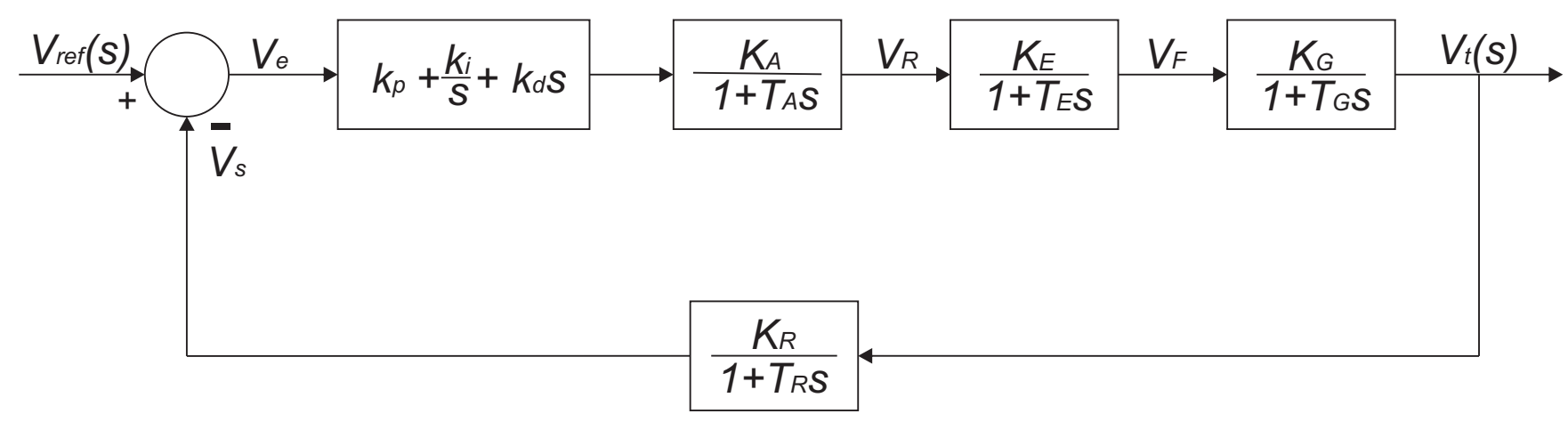

Figura 3. Diagrama de bloco do sistema AVR com PID.

Tabela 1. Parâmetros do sistema AVR.

\begin{tabular}{|c|c|c|}
\hline $\begin{array}{c}\text { Componente } \\
\text { do AVR }\end{array}$ & Ganho (K) & $\begin{array}{c}\text { Constante de } \\
\text { tempo }(\mathrm{T})\end{array}$ \\
\hline Amplificador & $K_{A}=10$ & $T_{A}=0.10 \mathrm{~s}$ \\
\hline Excitação & $K_{E}=1$ & $T_{E}=0.40 \mathrm{~s}$ \\
\hline Gerador & $K_{G}=1$ & $T_{G}=1.00 \mathrm{~s}$ \\
\hline Sensor & $K_{R}=1$ & $T_{R}=0.01 \mathrm{~s}$ \\
\hline
\end{tabular}

otimização do sistema (Ogata, 2003). A função objetivo utilizada para execução da Busca Tabu é proposta por Gaing (2004) e é definida em (6).

$$
f(x)=\frac{1}{\left(1-e^{-\beta}\right)\left(M_{p}+E_{s s}\right)+e^{-\beta}\left(t_{s}-t_{r}\right)}
$$

O parâmetro $\beta$ atua como um critério de ponderação entre os parâmetros $M_{p}$ e $E_{s s}$ com os tempos $t_{s}$ e $t_{r}$. Para um $\beta=0,7$ há um ponto de equilíbrio entre os parâmetros. No entanto, para valores maiores que 0,7 o algoritmo proporciona uma ênfase maior na redução do overshoot e do erro em regime permanente.

\subsection{Implementação da controle PID pela Busca Tabu}

A implementação do algoritmo de Busca Tabu para o ajuste ótimo do controlador PID em um sistema AVR foi realizado segundo os procedimentos descrito abaixo:

(a) Início;

(b) Definir a solução inicial $\left(s_{0}\right)$ e o número máximo de iterações $(\max I t)$. De início, $s_{0}$ será a solução global $s_{g}$, a função aspiração $(A)$ é dada pelo o valor da função objetivo de $s_{0}$ é a lista tabu $(T)$ e inicializada como conjunto vazio;

(c) Defina os limites dos parâmetros: $K_{p}, K_{i}$ e $K_{d}$;

(d) $i t=i t+1$;

(e) De maneira aleatória é gerado uma vizinhança $(V)$ de soluções deslocadas por um fator $m$ em torno da solução $s$ e é calculado o valor da função objetivo de cada vizinho, o melhor valor passará a ser $s^{\prime}$;

(f) Se a função objetivo de $f\left(s^{\prime}\right)>A$ $s_{g}=s^{\prime} ; A=f\left(s^{\prime}\right)$ é vá para $(i)$;

(g) Caso contrário, é verificado se $s^{\prime}$ pertence a $T$, se sim, essa solução é descartada e vá para o passo $(j)$; (h) Caso ele não esteja na lista tabu $(T)$ vá para $(i)$;

(i) É adicionado $s^{\prime}$ em $(T)$ e a solução $s$ passa a ser $s^{\prime}$;

(j) Verifica o critério de parada. Caso seja satisfeito, vá para o passo (k). Se não, volte o passo (d);

(k) Solução do algoritmo, $s_{g}$ e $f\left(s_{g}\right)$.

(1) Fim.

O fluxograma apresentado na Figura 4 resume o funcionamento da técnica de Busca Tabu aplicado para o controle da tensão terminal no sistema AVR.

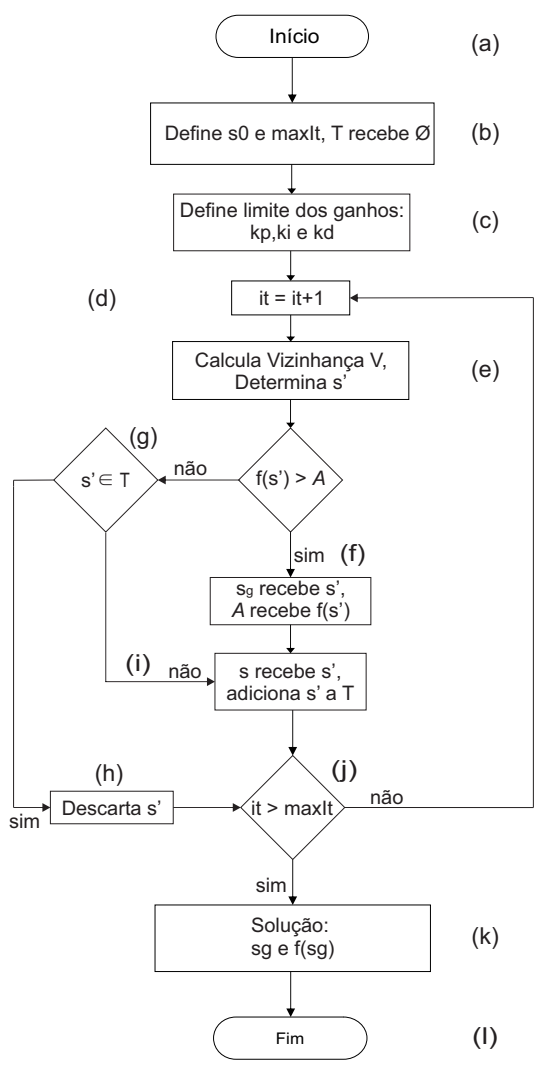

Figura 4. fluxograma da Busca Tabu. 


\section{RESULTADOS E SIMULAÇÕES}

Para o problema em estudo, utilizou-se o algoritmo descrita na Figura (4) e adotou-se os seguintes valores para os parâmetros da técnica de Busca Tabu: (i) $\left[\begin{array}{lll}0 & 0 & 0\end{array}\right] \leq$ $\left[\begin{array}{lll}K_{p} & K_{i} & K_{d}\end{array}\right] \leq\left[\begin{array}{lll}1,5 & 1,0 & 1,0\end{array}\right]$; (ii) $s_{0}=\left[\begin{array}{lll}0.75 & 0.50 & 0.50\end{array}\right]$; (iii) número de iterações igual a 100; (iv) os vizinhos são gerados de forma aleatória de maneira que seu movimento $(m)$ não ultrapasse um valor de 0,1 em módulo do valor da solução atual $(s)$; (v) a lista tabu $(T)$ é esvaziada a cada 10 iterações; (vi) o valor do parâmetro de ponderação da função objetivo $(\beta)$ é igual a 1,0 . O sistema AVR, como observado na Figura 3 foi implementado no ambiente do Simulink no MATLAB.

A fim de verificar o desempenho do método proposto para o sistema AVR foi aplicada uma entrada degrau unitário na tensão de referência $\left(V_{r e f}\right)$ do gerador síncrono, onde observou-se o controle de tensão terminal.

Em relação ao algoritmo de Busca Tabu, um ponto importante a ser determinado está relacionado com o número de vizinhos necessários para que a resposta do algoritmo apresente o melhor desempenho. Através da Tabela (2) observa-se o desempenho do sistema para variações do número de vizinhos, como forma de verificar a influência desse parâmetro é realizado 100 simulações para cada número de vizinhos apresentados.

Tabela 2. Variação da função objetivo em relação ao número de vizinhos.

\begin{tabular}{|c|c|c|c|c|}
\hline Número de & \multicolumn{4}{|c|}{ Função Objetivo } \\
\cline { 2 - 5 } Vizinhos & Maior & Menor & Média & $\begin{array}{c}\text { Desvio } \\
\text { Padrão }\end{array}$ \\
\hline 10 & 26,48 & 3,51 & 10,47 & 10,97 \\
\hline 25 & 26,86 & 3,71 & 17,24 & 8,62 \\
\hline 50 & 27,01 & 25,98 & 26,53 & 0,34 \\
\hline 75 & 27,00 & 26,34 & 26,76 & 0,19 \\
\hline 100 & 27,17 & 26,81 & 26,97 & 0,16 \\
\hline
\end{tabular}

Como verificado na Tabela (2), quanto maior o número de vizinhos melhor a qualidade das simulações, isso pode ser verificado através dos valores da média e desvio padrão da função objetivo para o caso de 100 vizinhos. Isso pode ser explicado pelo aumento do número de vizinhos, que consequentemente ocasiona um maior número de pontos a serem verificados. Como resultado, ocorre uma maior possibilidade que algum desses pontos convirjam para o ponto ótimo ou para um valor próximo.

A fim de enfatizar as vantagens da técnica proposta, foi comparado o resultado determinado por Busca Tabu com o número de vizinho igual a 100, com os valores encontrados pelas técnicas de PSO e GA realizado no trabalho de Gaing (2004). A Tabela (3) apresenta os valores do controlador PID determinados pelas técnicas de Busca Tabu, PSO e GA.

Tabela 3. Parâmetros do PID determinados por diferente técnicas.

\begin{tabular}{|c|c|c|c|}
\hline Técnica & $K_{p}$ & $K_{i}$ & $K_{d}$ \\
\hline Busca Tabu & 0,6682 & 0,4653 & 0,2700 \\
\hline PSO & 0,6751 & 0,5980 & 0,2630 \\
\hline GA & 0,7722 & 0,7201 & 0,3196 \\
\hline
\end{tabular}

Na Figura 5 verifica-se o desempenho dinâmico da resposta da tensão terminal para uma entrada degrau unitário na tensão de referência do sistema AVR para os parâmetros determinados na Tabela (3).

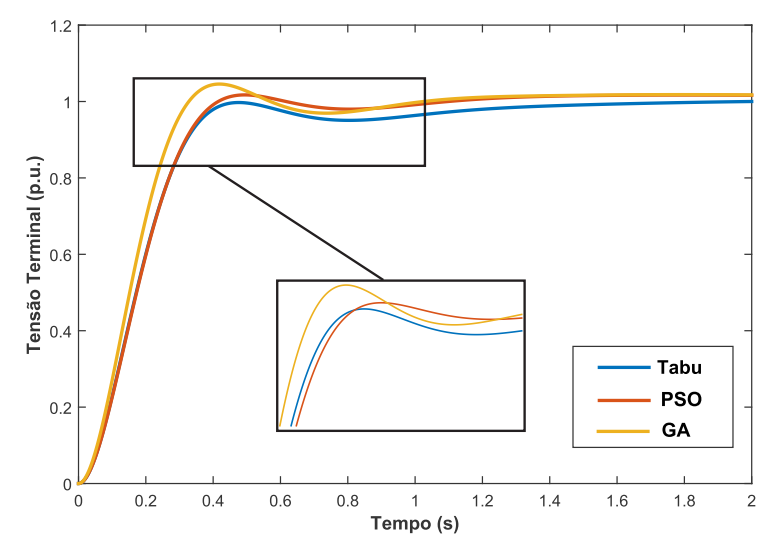

Figura 5. Desempenho da Tensão terminal para uma entrada degrau unitário na tensão de referência.

Analisando a Figura 5 pode-se concluir que a técnica de Busca Tabu proporcionou o melhor ajuste do controlador PID, tendo em vista que a sua resposta permitiu um valor de overshoot e de erro em regime permanente praticamente zero e também uma menor diferença entre os tempos de acomodação e de subida quando comparado com as outras técnicas. A Tabela 4 mostra uma comparação entre os principais índices da resposta temporal obtidos através da Figura 5 para os métodos de Busca Tabu, PSO e GA.

Tabela 4. Comparação entre os índices para os métodos de Busca Tabu, PSO e GA.

\begin{tabular}{|c|c|c|c|c|}
\hline Técnica & $M_{p}(\%)$ & $E_{s s}(\%)$ & $t_{s}(s)$ & $t_{r}(s)$ \\
\hline $\begin{array}{c}\text { Busca } \\
\text { Tabu }\end{array}$ & 0,00 & 0,0006 & 0,3563 & 0,2563 \\
\hline PSO & 1,71 & 1,6300 & 0,3795 & 0,2648 \\
\hline GA & 4,54 & 1,7300 & 0,8645 & 0,2138 \\
\hline
\end{tabular}

\section{CONCLUSÃO}

No presente artigo, o ajuste ideal do controlador PID é realizado pela técnica de Busca Tabu. A resposta otimizada da tensão terminal do gerador para uma entrada degrau unitário realizada pelo método proposto é comparada com a resposta obtida pelas técnicas de PSO e GA. Os resultados da simulação demonstram que a técnica de Busca Tabu é mais eficiente para ajustar os parâmetros do PID. Essa proporciona uma melhora na eficácia do sistema AVR e também nas características de resposta, tais como tempo de estabilização, amplitude de pico, tempo de subida e no erro de controle da tensão.

\section{AGRADECIMENTOS}

Os autores agradecem ao Grupo de Pesquisa em Inteligência Computacional Aplicada (GICA) do Departamento de Engenharia Elétrica da Universidade Federal da Paraíba. 


\section{REFERÊNCIAS}

Areerak, K. and Sujitjorn, S. (2002). Performance comparison between genetic algorithm and tabu search methods. Suranaree J. Sci. Technol, 9(1), 61-68.

Elgerd, O.I. (1982). Electric energy systems theory: an introduction.

Farouk, N. and Bingqi, T. (2012). Application of selftuning fuzzy pid controller on the avr system. In 2012 IEEE International Conference on Mechatronics and Automation, 2510-2514. IEEE.

Finch, J., Zachariah, K., and Farsi, M. (1999). Turbogenerator self-tuning automatic voltage regulator. IEEE Transactions on Energy Conversion, 14(3), 843-848.

Gaing, Z.L. (2004). A particle swarm optimization approach for optimum design of pid controller in avr system. IEEE transactions on energy conversion, 19(2), 384391.

Gaspar-Cunha, A., Takahashi, R., and Antunes, C.H. (2012). Manual de computação evolutiva e metaheurística. Imprensa da Universidade de Coimbra/Coimbra University Press.

Glover, F. (1977). Heuristics for integer programming using surrogate constraints. Decision sciences, 8(1), 156-166.

Glover, F. (1986). Future paths for integer programming and links to artificial intelligence. Computers \& operations research, 13(5), 533-549.

Glover, F. (1989). Tabu search part 1. ORSA Journal on computing, 1(3), 190-206.

Gözde, H., Taplamacioğlu, M.C., and Ari, M. (2017). Simulation study for global neighborhood algorithm based optimal automatic voltage regulator (avr) system. In 2017 5th International Istanbul Smart Grid and Cities Congress and Fair (ICSG), 46-50. IEEE.

Hansen, P. (1986). The steepest ascent mildest descent heuristic for combinatorial programming. In Congress on numerical methods in combinatorial optimization, Capri, Italy, 70-145.

Kundur, P., Balu, N.J., and Lauby, M.G. (1994). Power system stability and control, volume 7. McGraw-hill New York

Lahcene, R., Abdeldjalil, S., and Aissa, K. (2017). Optimal tuning of fractional order pid controller for avr system using simulated annealing optimization algorithm. In 2017 5th International Conference on Electrical Engineering-Boumerdes (ICEE-B), 1-6. IEEE.

Ogata, K. (2003). Engenharia de controle moderno, ed. São Paulo: $4^{\circ}$.

Swidenbank, E., Brown, M., and Flynn, D. (1999). Selftuning turbine generator control for power plant. $\mathrm{Me}$ chatronics, 9(5), 513-537.

Thainiam, P. (2018). Developing tabu search with intensification and diversification for the seriation problem. In 2018 5th International Conference on Industrial Engineering and Applications (ICIEA), 279-283. IEEE. 